\title{
BMJ Open Longitudinal assessment of chlorpyrifos exposure and self-reported neurological symptoms in adolescent pesticide applicators
}

\author{
Khalid Khan, ${ }^{1}$ Ahmed A Ismail, ${ }^{2}$ Gaafar Abdel Rasoul, ${ }^{2}$ Matthew R Bonner, ${ }^{3}$ \\ Michael R Lasarev, ${ }^{4}$ Olfat Hendy, ${ }^{5}$ Manal Al-Batanony, ${ }^{2}$ Alice L Crane, ${ }^{6}$ \\ Steven T Singleton, ${ }^{6}$ James R Olson, ${ }^{3,6}$ Diane S Rohlman ${ }^{1,4}$
}

To cite: Khan K, Ismail AA, Abdel Rasoul G, et al. Longitudinal assessment of chlorpyrifos exposure and self-reported neurological symptoms in adolescent pesticide applicators. BMJ Open 2014;4:e004177. doi:10.1136/bmjopen-2013004177

- Prepublication history and additional material for this paper is available online. To view these files please visit the journal online (http://dx.doi.org/10.1136/ bmjopen-2013-004177).

Received 5 October 2013 Revised 11 December 2013 Accepted 10 January 2014

CrossMark

For numbered affiliations see end of article.

Correspondence to Dr Diane S Rohlman; diane-rohlman@uiowa.edu

\section{ABSTRACT}

Objectives: Occupational exposure of organophosphorus pesticides, such as chlorpyrifos (CPF), in adolescents is of particular concern because of the potential vulnerability of the developing neurological system. The objectives of this study were to examine how neurological symptoms reported over the application season vary across time, whether these effects are reversible postapplication and if there are associations between CPF biomarkers and neurological symptoms in an adolescent study population.

Setting: The longitudinal study was conducted in two agricultural districts of Menoufia Governorate, Egypt between April 2010 and January 2011.

Participants: Male adolescent participants, including CPF applicators $(n=57)$ and non-applicators $(n=38)$, were recruited.

Primary and secondary outcome measures: Selfreported data for 25 neurological symptoms were collected at 32 time points over the 8-month period before, during and after the application season. Additionally, urine and blood samples were collected to measure urine trichloro-2-pyridinol (TCPy), a CPFspecific biomarker and blood cholinesterase activity.

Results: Applicators and non-applicators report the highest numbers of symptoms during the application season, followed by a reduction in symptoms after the application ended. Applicators reported a greater percentage of neurological symptoms, relative to baseline, than non-applicators after accounting for potential covariates. Among the applicators, cumulative TCPy was positively and significantly associated with the average percentage of symptoms ( $\mathrm{B}=4.56,95 \% \mathrm{Cl} 3.29$ to 5.84 ; $p<0.001)$. Significant associations $(p=0.03-0.07)$ between the change in butyrylcholinesterase activity from the preapplication to the postapplication season and several domains of neurological symptoms were also found, even after adjusting for potential covariates.

Conclusions: These observations demonstrate changes in the reporting of symptoms across the application season, showing an increase in symptom reporting during application and recovery following the end of pesticide application. These findings reinforce the growing concern regarding the neurotoxic health effects of CPF in

\section{Strengths and limitations of this study}

- This is the first longitudinal study demonstrating an association between chlorpyrifos (CPF) exposure and reporting of neurological symptoms in adolescent applicators.

- The study is also novel in its approach in that it includes prospective measures of biomarkers of CPF exposure and effect and examines their associations with neurological symptoms.

- The non-specific nature of many of the symptoms is a limitation of the current study.

- The small sample size of this study may have influenced the significance levels of the exposure-outcome relationships.

adolescent applicators in developing countries and the need for developing and implementing intervention programmes.

\section{INTRODUCTION}

The high use of organophosphorus pesticides (OPs) has been recognised as a major global public health challenge for agriculture-based communities, due to their associations with adverse neurological outcomes. Immediate and short-term neurological signs and symptoms ranging from less severe (headache, dizziness, nausea, etc) to more severe (muscle weakness, bronchospasm, change in heart rate, etc) have all been reported after occupational exposure to OPs. ${ }^{2}$ Although high levels of occupational OP exposure can be associated with symptoms persisting for several years, ${ }^{3}$ repeated moderate-to-low exposures can also produce chronic neurological symptoms and deficits in neurobehavioural performance. ${ }^{4}$ Converging evidence regarding the 
associations between OP exposures and neurological symptoms is based on occupational studies with adults conducted in a wide range of settings, including comparisons between exposed and non-exposed farm workers in the USA, ${ }^{5}$ South Africa, ${ }^{6}$ Nicaragua, ${ }^{7} 8$ Kenya, ${ }^{9}$ Sri Lanka ${ }^{10}$ and Egypt. ${ }^{11}$ Additional evidence for the effect of pesticides on somatic and mood symptoms is also found in the literature. ${ }^{2} 12$

Although it is illegal, there have been reports of involvement of US adolescents in mixing and applying pesticides in some agricultural communities. ${ }^{13}{ }^{14}$ The developing bodies of children and adolescents may not break down pesticide as effectively as those of adults and they may receive a larger dose per unit of body weight for a given exposure due to their smaller body size, ${ }^{15}$ making them more vulnerable to neurological effects. Animal and human studies have also suggested that paraoxonase-1 (PON-1), an organophosphate detoxifying enzyme, is less active in younger populations, making them more vulnerable to OP toxicity. ${ }^{16}{ }^{17}$ A recent study has found an association of environmental chlorpyrifos (CPF) exposure with structural changes in the developing brain of children and adolescents. ${ }^{18}$ In developing countries, children and adolescents are engaged in risky agricultural activities including the application of OPs. ${ }^{19}$ In two epidemiological studies, Egyptian and Indian children and adolescents living in agricultural communities have demonstrated associations between occupational and environmental OP exposure and neurological and neuromuscular problems. ${ }^{20-22}$

Biomarkers have been used to characterise OP exposure in epidemiological and occupational studies. Urinary trichloro-2-pyridinol (TCPy) is a relatively specific metabolite of CPF exposure, eliminated in the urine with a half-life of $27 \mathrm{~h}$ following exposure. ${ }^{23}$ Owing to the ease and non-invasiveness of the collection of urine samples, TCPy is widely recognised as a useful biomarker of exposure, particularly in children and adolescents. ${ }^{24} 25$ The classic mode of OP toxicity is manifested by the inhibition of cholinesterase (ChE). Blood acetylcholinesterase (AChE) and butyrylcholinesterase (BChE) are biomarkers of effect, with BChE being more sensitive to inhibition by OP pesticides. ${ }^{26} \mathrm{~A}$ small number of adult studies found associations between inhibition of cholinergic activities with self-reported symptoms ${ }^{9}{ }^{10}$; however, this relationship has rarely been examined in adolescent studies.

Understanding the relationship between OP exposure and the change in neurological symptoms over time (temporal change) is important because applicationrelated exposure follows a seasonal pattern in most areas. Two longitudinal studies with agricultural workers demonstrated that short-term neurological signs and symptoms were associated with initial acute episodes of exposure, which eventually advanced into long-term sequelae. ${ }^{7}{ }^{12}$ However, these studies did not characterise exposure by identifying specific types of OPs that were related to the symptoms.
The primary objective of this study was to determine whether occupational exposure to CPF is associated with self-reported neurological symptoms in adolescents. Through a prospective study, we compared adolescent applicators exposed to CPF with adolescent nonapplicators working and residing in agricultural communities in Egypt. Typically, CPF is the primary insecticide used by pesticide applicators in Egyptian cotton fields, offering us a unique exposure opportunity with wellcharacterised occupational exposure. The possibility of potential confounding effects of other neurotoxic pesticides was minimal because of the limited use of other pesticides in the study area. The goals of the study were to examine how neurological symptoms vary over time during the exposure season, if these effects could reverse at the cessation of exposure and whether there are any associations between CPF biomarkers and neurological symptoms in the adolescent study population.

\section{METHODS}

\section{Study area and population}

Two agricultural districts were selected from Menoufia Governorate, Egypt (see online supplementary figure S1) to conduct a prospective study from April 2010 to January 2011. In Egypt, adolescents are hired seasonally to apply pesticides to cotton fields and the schedule of pesticide applications to the cotton crop is regulated by the Ministry of Agriculture. The typical workday was from 8:00 to 12:00 and from 15:00 to 19:00, 6 days/week. During 2010, approximately $2100 \mathrm{~L}$ of OPs were applied to 5700 acres of cotton fields (personal communication with the Ministry of Agriculture). CPF is the primary OP applied to the cotton crop from mid-June to early August. Although there are slight variations in the timing of CPF application between the two districts (figure 1), the application patterns are consistent across these two areas. As there is no regulation in Egypt for the mandatory use of personal protective equipment, dermal exposure and inhalation were considered as the potential route of exposure in this population. ${ }^{20}$ Recently, Fenske et $a l^{27}$ reported that dermal exposure and subsequent absorption through the skin accounted for $94-96 \%$ of the total dose of CPF in Egyptian pesticide applicators.

\section{Recruitment and data collection}

Fifty-eight male adolescents, aged 12-21 years, who were hired seasonally by the Ministry of Agriculture to spray pesticides in the cotton fields, were recruited from two districts in the Menoufia Governorate. Forty adolescent non-applicators were recruited through convenience sampling (ie, word of mouth, direct communication utilising contacts through the staff from the local Ministry of Agriculture) from the same districts as the applicators for the cotton crop. These adolescents never worked in the cotton fields as pesticide applicators. One adolescent was excluded from the final analysis due to his inconsistency in participating in the study activities and two other 


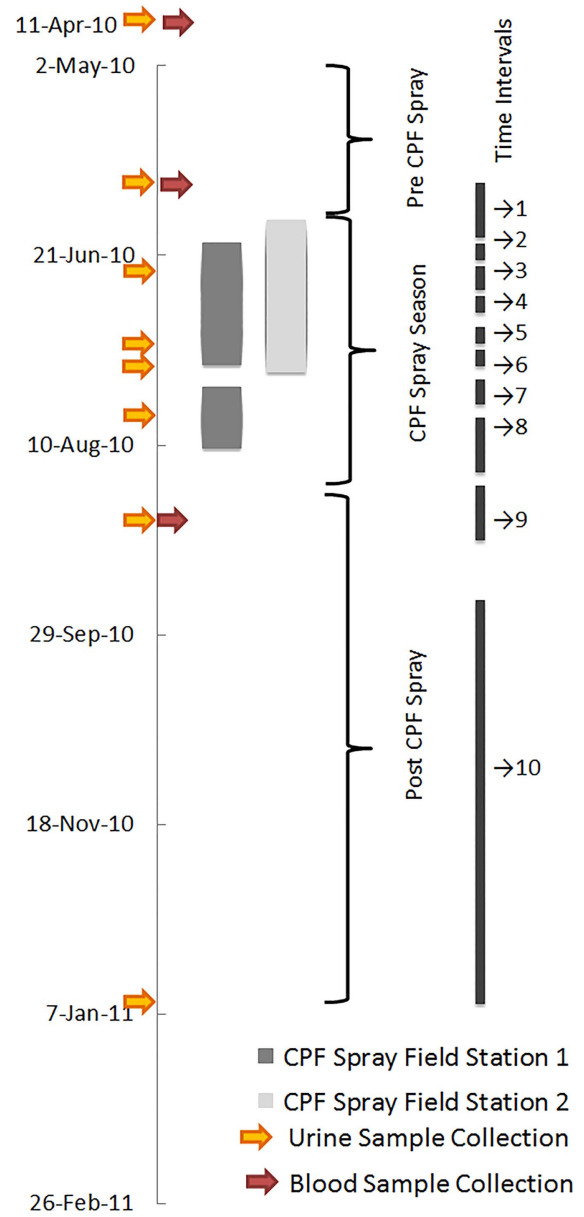

Figure 1 Chlorpyrifos (CPF) application in the study area showing time intervals in field stations 1 and 2 .

participants were excluded for questionable sample integrity, resulting in a final sample size of 95 (57 applicators and 38 non-applicators). Written informed consent was obtained from all participants and their legal guardian (for those under 18). All the participants were monetarily compensated for their time during the questionnaire survey and biological samples ( US $\$ 5$ per visit).

Data collection, for both applicators and nonapplicators, occurred at the primary field station for each district. Pesticide applicators and supervisors meet in the field stations, which also provide storage area for the pesticides and the equipment used for application.

\section{Outcome assessment}

We developed a 25-item, short-term neurological symptom questionnaire on the basis of the widely used Q16 questionnaire ${ }^{28}$ and a modified version of the Q16 used in a previous study on licensed pesticide applicators. ${ }^{29}$ The 25 symptoms were grouped into six domains: behavioural, autonomic, cognitive, sensory, motor and non-specific temporary disability (table 1 ). The questionnaire had five response options (0-4) for each symptom ranging from 'never' (coded as 0 ) to 'everyday of the

\begin{tabular}{|c|c|}
\hline Domains & Symptoms \\
\hline Behavioural symptoms & $\begin{array}{l}\text { 1. Tense or anxious }{ }^{\star} \\
\text { 2. Excessively angry or } \\
\text { irritable }{ }^{*} \dagger \\
\text { 3. Depressed or withdrawn }{ }^{*} \dagger\end{array}$ \\
\hline Autonomic symptoms & $\begin{array}{l}\text { 4. Nausea* } \\
\text { 5. Heavy sweating* } \dagger \\
\text { 6. Loss of appetite* } \\
\text { 7. Fast heart rate }{ }^{*} \dagger \\
\text { 8. Excessive salivation }\end{array}$ \\
\hline Cognitive symptoms & $\begin{array}{l}\text { 9. Difficulty concentrating }{ }^{*} \dagger \\
\text { 10. Being absentminded and } \\
\text { memory problem }{ }^{\star} \dagger\end{array}$ \\
\hline Sensory symptoms & $\begin{array}{l}\text { 11. Difficulty seeing at night } \\
\text { 12. Blurred or double vision } \\
\text { 13. Numbness in hands and } \\
\text { feet }^{*} \\
\text { 14. Sense of smell or taste } \\
\text { change }^{*} \\
\text { 15. Ringing in ears }\end{array}$ \\
\hline Motor symptoms & $\begin{array}{l}\text { 16. Difficulty with balance } \\
\text { 17. Weakness in arms and } \\
\text { legs }^{*} \\
\text { 18. Involuntary movement of } \\
\text { arms and legs } \\
\text { 19. Shaking in hands } \dagger \\
\text { 20. Difficulty speaking* }\end{array}$ \\
\hline $\begin{array}{l}\text { Temporary disability } \\
\text { (non-specific symptoms) }\end{array}$ & $\begin{array}{l}\text { 21. Dizziness }{ }^{*} \\
\text { 22. Headache }{ }^{*} \dagger \\
\text { 23. Momentary loss of } \\
\text { consciousness }^{*} \\
\text { 24. Fatigue }{ }^{*} \dagger \\
\text { 25. Insomnia }\end{array}$ \\
\hline
\end{tabular}

week' (coded as 4). Since more than $90 \%$ of the responses were between $0-2$ (1, once a week and 2, once in every 2-3 days), we recoded each of the symptom responses to ' 0 ' or 'never' and ' 1 ' or 'at least once a week or more'. Self-reported neurological symptom counts were collected at 32 irregularly spaced dates over an 8-month period from early June 2010 through early January 2011. These time points ranged across three different time periods: preapplication, application and postapplication. For each time point, the number of positive responses (a response was considered positive and coded as ' 1 ' when the participant reported the frequency of the symptom 'at least once a week or more') was totalled for each person to yield a score ranging from 0 to 25 ; division by 25 produced the proportion of symptoms endorsed at each of the 32 time points. This outcome variable was used to compare the change of symptoms over time between applicators and nonapplicators. All these time points were collapsed into 10 separate non-overlapping intervals lasting between 1 and 4 weeks in length (figure 1). Symptom data during the 
preapplication period, including the first 15 days of the study, were collectively taken to represent the baseline time interval (or time interval 1). Symptom reporting from the other nine remaining time intervals was evaluated against time interval 1 . The next five time intervals, between 19 June and 21 July, were during the application period of CPF. The remaining four time intervals occurred between 24 July 2010 and 5 January 2011 and reflect the postapplication period, although a brief CPF application was reported in the district where field station 1 was located. The proportions of symptoms over all the 32 time points were averaged to produce a season-level average percentage of neurological symptoms over the entire study period. This outcome variable was used to examine the relationships between the biomarkers (TCPy, AChE and BChE) and symptoms. Participants also completed a questionnaire during baseline addressing their sociodemographic status, household and occupational use of pesticides.

\section{Urine collection and analysis}

Spot urine samples were collected in new and individually wrapped cups at the beginning of the work shift at eight time points between April 2010 and January 2011. The cups were opened at the time of sample collection. Urine samples were subsequently transferred to the laboratory at Menoufia University in a cooler with wet ice. At the laboratory, $4 \mathrm{~mL}$ aliquots of urine were transferred into labelled $5 \mathrm{~mL}$ cryovials within hours of sampling and stored at $-20^{\circ} \mathrm{C}$. The banked urine samples were express mailed on dry ice to the University of Buffalo laboratory for analysis of pesticide metabolites; duplicate samples were retained in the $-20^{\circ} \mathrm{C}$ freezer at Menoufia University. Urine samples in field station 2 were collected 1 day after the collection date of field station 1.

Creatinine concentrations were measured using the Jaffe reaction. ${ }^{30}$ The method of urinary TCPy measurement (a primary metabolite of CPF) has been described elsewhere. ${ }^{24}$ Briefly, samples were analysed using gas chromatography-mass spectrometry (negative-ion chemical ionisation) and utilised 13C-15N-3,5,6-TCPy as an internal standard. Samples were hydrolysed with hydrochloric acid, extracted with toluene and derivatised using N-(tert-butyldimethylsilyl)-N-methyltrifluoro-acetamide (Sigma Aldrich, USA). A spiked quality control (QC) sample was routinely run with the analytical samples and the metabolite concentration was determined from a standard curve for the peak area for the selective ion. The QC samples consisted of lab samples that were first analysed for TCPy and the levels were non-detectable. The TCPy standard curve was linear from 1 to $200 \mathrm{ng} / \mathrm{mL}$ with a correlation coefficient of 1.000. Samples spiked with $50 \mathrm{ng}$ of TCPy $/ \mathrm{mL} \quad(\mathrm{n}=20)$ gave an average metabolite recovery of $94.8 \%$ (range 92-98\%; SD=0.931; relative SD, $\mathrm{RSD} \%=1.965$ ). A $1 \mathrm{ng}$ $\mathrm{TCPy} / \mathrm{mL}$ spiked sample was run 10 times and the within series $\mathrm{RSD} \%=1.6$. The minimum detection level was $0.5 \mathrm{ng} / \mathrm{mL}$ of urine.

\section{Blood collection and ChE analysis}

To establish the baseline ChE activity, preapplication blood draws occurred on 11 April and 2 June 2010, prior to the start of the official government-regulated CPF application season. As with the urine collection, blood draws occurred in field station 21 day later. Changes in AChE and BChE levels from baseline to the end of the CPF-application season (blood collected on 4 September 2010) were estimated. Blood samples were collected by venipuncture into $10 \mathrm{~mL}$ lavender top (EDTA) vacutainer tubes and immediately placed on wet ice and transported to Menoufia University, where they were analysed in duplicate for AChE and BChE activity using an EQM Test-Mate kit (EQM Research Inc, Cincinnati, Ohio, USA) as described previously. ${ }^{24}$

\section{Statistical analysis}

We used SPSS V.18.0 and STATA (V.11; Stata Corporation, College Station, Texas, USA) for the statistical analysis. Sociodemographic variables were summarised and described using means and SDs for continuous responses and percentages for discrete outcomes; simple comparisons between applicators and non-applicators were completed using t tests or $\chi^{2}$ tests. To calculate the value of cumulative TCPy for each participant, we used STATA's pharmacokinetic function (pkexamine) to employing the trapezoid rule to estimate the area under the curve for each participant over the study time. By definition, cumulative TCPy was the sum of the concentration at each time point multiplied by the duration between time points. This variable reflects the total amount of TCPy excreted over the study period for which urine was collected and assayed. Concentrations of cumulative TCPy, $\mathrm{AChE}$ and $\mathrm{BChE}$ exhibited pronounced right skewed distribution and more than a threefold separation between the minimum and maximum observed values; consequently, these responses were log-transformed prior to analysis to improve symmetry. AChE and BChE were expressed as a log-transformed ratio of postapplication activity relative to preapplication activity. Then the associations between the change of these $\mathrm{ChE}$ markers from preapplication to postapplication seasons and selfreported symptoms were examined using linear regression models that took potential covariates into account. Similar regression analyses were used to examine the relationship between cumulative TCPy and neurological symptoms. All $\mathrm{p}$ values are two sided with significance judged relative to a 0.05 level.

Spearman correlation coefficients were used to estimate associations between urine and blood biomarkers and symptom scores. Generalised estimating equations $(\mathrm{GEE})^{31}$ were used to model the proportion of neurological symptoms reported in each time interval while controlling for the number of days worked (within 5 days of the symptom reporting date), home use of 
pesticides, age, education and income levels. The one fitted model was used to estimate changes over time, relative to the first time interval (2 June-16 June), for applicators and non-applicators, as well as to examine whether changes relative to baseline differed between the two groups (via group-by-time interaction).

\section{RESULTS}

\section{Sociodemographic characteristics}

Ninety-two of the participants (97\%) were between 12 and 18 years with the remaining three between 19 and 21. The two groups, non-applicators and applicators, did not differ significantly in terms of age, educational status, family income, number of people in house, years of pesticide use at home, and insecticide and rodenticide use at home (table 2). Compared to nonapplicators, a significantly higher number of applicators lived close to the field (within $25 \mathrm{~m}$ ), had carpets in their homes and applied herbicides at home. Applicators had a significantly lower body mass index than non-applicators. On average, applicators had been working in the field for a little over 3 years.

\section{Change in symptoms over time}

We considered days $0-14$ as the baseline time interval (time interval 1) when no application of CPF was reported. While examining symptoms reported over time among applicators and non-applicators, we took various potential confounders into account. These include occupational factors such as days worked per week in pesticide applications, number of years of pesticide use at home and sociodemographic factors such as age, education and income level of the participants. Applicators began increased reporting of neurological symptoms at the beginning of the CPF application season (at time interval 2 between days 17-21 of the study). The percentage of neurological symptoms continued to increase during the application season and reached the peak at time interval 6 , representing days 45-48, the time when the CPF application period ended. This was followed by a drop in symptom reporting indicating a small recovery due to the cessation of exposure in both districts. The highest peak in symptom reporting was observed at time interval 8 representing days 63-77 (table 3). This happened perhaps due to a small episode of CPF application in field station 1 (between time intervals 7 and 8). Similar to the applicators, the non-applicators also demonstrated the highest increase in the proportion of neurological symptoms during time interval 8 , although the magnitude of the change was smaller (14 percentage point increase of symptoms relative to baseline interval). The change of neurological symptoms relative to baseline declined over the next two time intervals (9 and 10) in both groups, indicating a recovery phase during postapplication. For applicators, the percentage of reported symptoms at each of the nine subsequent time intervals was always higher than the percentage observed at baseline; nonapplicators, by contrast, had a pattern of reported

Table 2 Sociodemographic characteristics for participants at baseline

\begin{tabular}{|c|c|c|}
\hline Variables & Non-applicators $(n=38)$ mean (SD) & Applicators $(n=57)$ mean $(S D)$ \\
\hline Age & $16.6(2.4)$ & $16.2(1.6)$ \\
\hline Education & $9.8(1.8)$ & $9.9(1.8)$ \\
\hline Height $(\mathrm{cm})^{*}$ & $166.3(12.0)$ & $163.4(10.0)$ \\
\hline Weight $(\mathrm{kg}) \dagger$ & $62.0(15.4)$ & $54.2(8.6)$ \\
\hline BMI $\left(\mathrm{kg} / \mathrm{m}^{2}\right) \dagger$ & $22.1(3.7)$ & $20.2(2.2)$ \\
\hline Number of people in house & $5.6(1.1)$ & $6.0(1.8)$ \\
\hline Home pesticide use (years)†‡ & $1.6(1.9)$ & $2.5(1.9)$ \\
\hline Occupational application of pesticides (years) & - & $3.1(1.5)$ \\
\hline Days/week of pesticide application & - & $4.8(1.3)$ \\
\hline \multirow[t]{2}{*}{ Hours/day of pesticide application } & - & $5.2(0.7)$ \\
\hline & Per cent $(n)$ & Per cent $(n)$ \\
\hline Family monthly income (<500 E) & $78.9(30)$ & $71.9(41)$ \\
\hline Applied pesticides in home in past 5 years (yes) $†$ & $47.4(18)$ & $78.9(45)$ \\
\hline Computer use (once a week or more) $†$ & $65.8(25)$ & $45.6(26)$ \\
\hline Carpet in house (yes) $\dagger$ & $27.0(10)$ & $54.4(31)$ \\
\hline Live within $25 \mathrm{~m}$ to agricultural field (yes) $\dagger$ & $23.7(9)$ & $50.9(29)$ \\
\hline \multicolumn{3}{|l|}{ Types of pesticides applied at homeq } \\
\hline Herbicides $†$ & $13.0(3)$ & $44.9(21)$ \\
\hline Insecticides & $83.3(20)$ & $93.9(46)$ \\
\hline Rodenticides & $16.7(4)$ & $14.3(7)$ \\
\hline
\end{tabular}




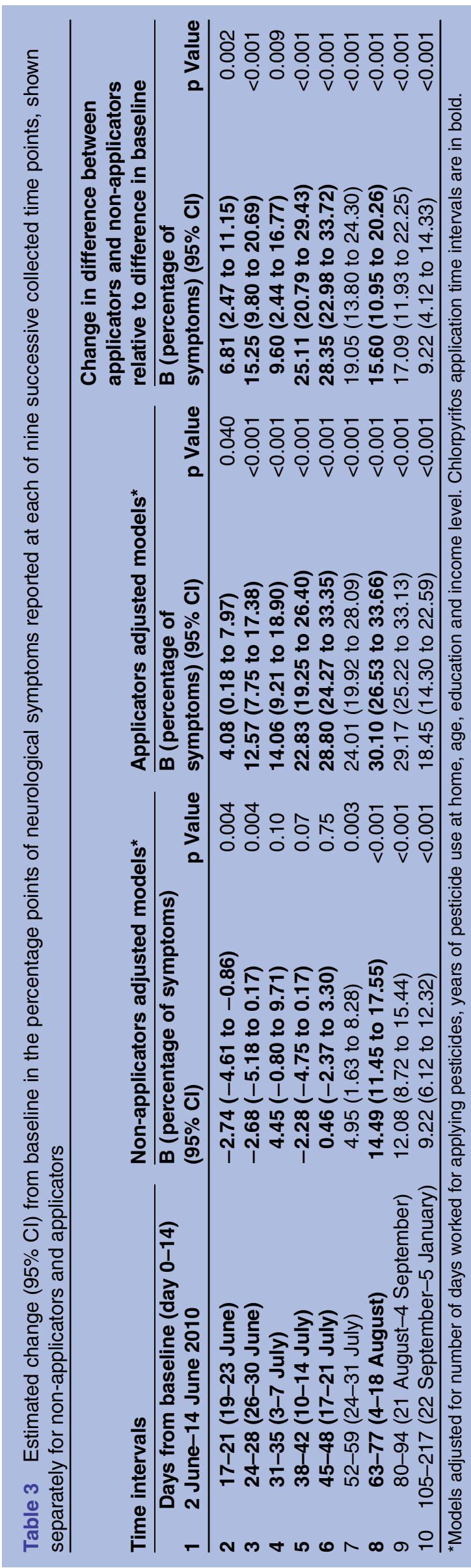

symptoms that increased and decreased relative to the baseline time interval (preapplication) over the course of the study (figure 2).

When applicators and non-applicators are compared with respect to change in percentage of symptoms (relative to baseline), it was always the case that the change (percentage point change relative to baseline) for applicators was greater than the corresponding change for non-applicators even after adjusting for the covariates (table 3).

Associations of neurological symptoms with biomarkers TCPy was detected in $100 \%$ of the samples. Summary statistics for TCPy, AChE and BChE of the study samples have already been reported by Crane $e t a l^{32}$ Mean creatinine concentration of the urine samples was reported to be $1696 \mu \mathrm{g} / \mathrm{mL}$ with a maximum of 4199 and a minimum of $164 \mu \mathrm{g} / \mathrm{mL}$. In brief, the applicators had a much higher mean and estimated median peak TCPy concentration than the non-applicators (mean 719 vs $44.9 \mu \mathrm{g} / \mathrm{g}$ creatinine; estimated median 137 vs $19.7 \mu \mathrm{g} / \mathrm{g}$ creatinine). In our study sample, BChE was found to be more sensitive to CPF exposure than AChE, with median activity reduced by $37 \%$ from baseline in applicators and $13 \%$ in non-applicators during the CPF application period.

A scatter plot of cumulative TCPy $(\mu \mathrm{g} / \mathrm{g}$ creatinine) against average percentage points of symptoms revealed distinct exposure-response gradients by pesticide application status (applicators vs non-applicators; figure 3).

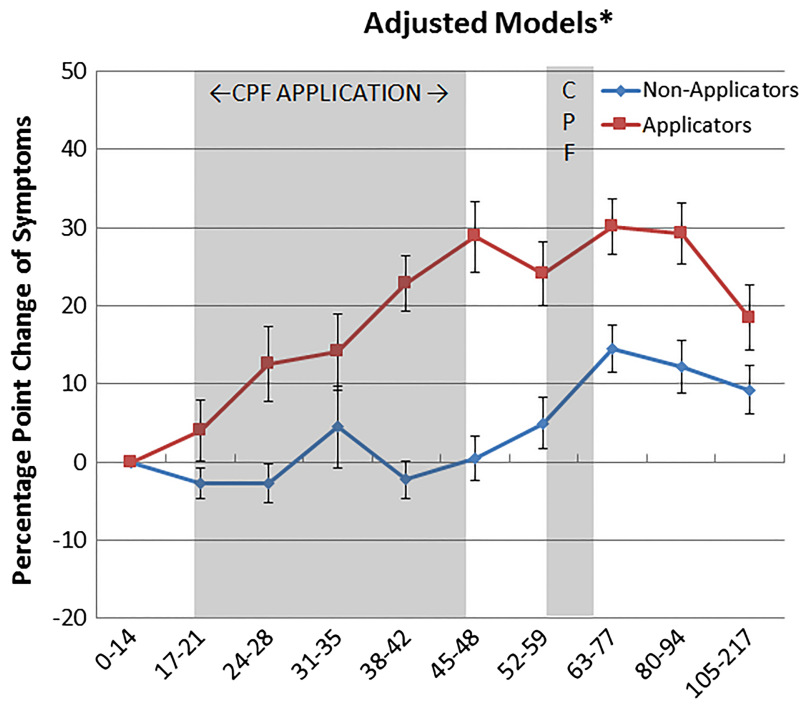

Time Intervals (Days from Baseline)

Figure 2 Difference, relative to baseline, in the percentage of symptoms reported at each of nine subsequent time intervals; error bars represent $95 \%$ confidence limits for the difference. *models adjusted for number of days worked for applying pesticides, years of pesticide use at home, age, education and family monthly income. Chlorpyrifos (CPF) application time intervals are shaded in grey. 


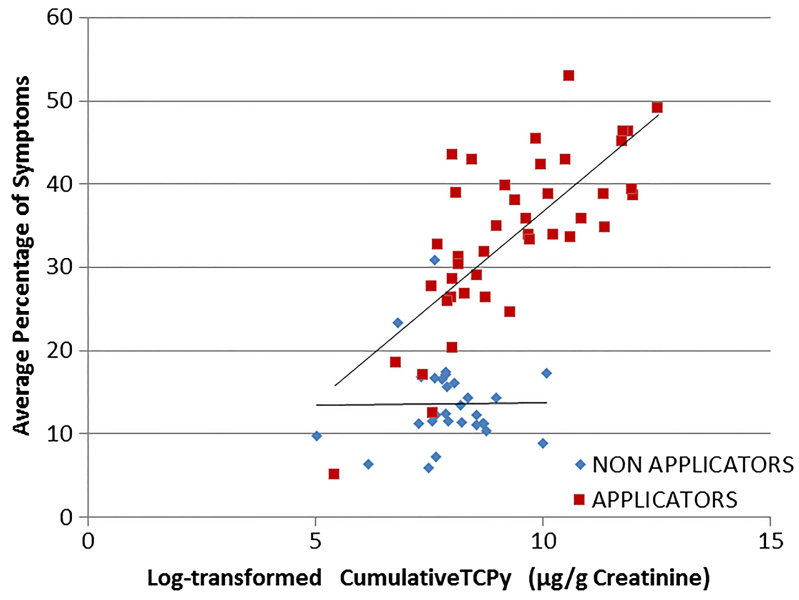

Figure 3 Scatter plots of cumulative trichloro-2-pyridinol (TCPy) ( $\mu \mathrm{g} / \mathrm{g}$ creatinine) against percentage of symptoms $(n=70)$.

In addition, two other scatter plots of change in AChE activity and change in BChE activity from preapplication to postapplication against percentage of symptoms also revealed effect measure modification by pesticide application status (see online supplementary figures S2A,B). Therefore, separate linear models for applicators and non-applicators were used to examine the associations of these three biomarkers with symptoms.

Log-transformed TCPy was positively associated with the average percentage of neurological symptoms in the regression models after adjusting for other covariates that may confound the exposure-outcome relationship such as field stations, age, family monthly income, pesticide use at home and average number of hours worked in the field among applicators $(\mathrm{b}=2.68, \mathrm{p}=0.007)$. However, non-applicators demonstrated positive, but statistically non-significant, associations between TCPy and symptoms. Among applicators, AChE and BChE activity was negatively and significantly associated with the average percentage of neurological symptoms in the unadjusted models. In the adjusted models, these associations remained negative but became non-significant (table 4).

When we examined the biomarker-symptom relationship by domains of symptoms among the applicators, we observed significant positive associations of logtransformed TCPy with behavioural, autonomic, cognitive, motor and sensory problems after accounting for sociodemographic and occupational covariates (table 5). The magnitudes of associations (adjusted $\beta s$ ) were greater for autonomic, cognitive and sensory symptoms than in the other two domains. Although the logtransformed change in AChE activity was not associated with any of these subclasses, change in BChE activity demonstrated a significant association with average percentage of behavioural symptoms $(p=0.04)$ and a marginally significant association with average percentage of cognitive symptoms $(\mathrm{p}=0.07)$.

\section{DISCUSSION}

The self-reported symptom questionnaire has been globally recognised as the primary method to capture symptom data in exposed populations. The most common questionnaire utilised is the extended or modified versions of Q16, ${ }^{28}$ which has been used in many international studies, including a study with Nicaraguans living close to cotton fields, ${ }^{8}$ Sri Lankan farm workers ${ }^{10}$ and Colorado agricultural communities. ${ }^{33}$ However, time intervals between exposure and collection of symptom data in these studies varied from 1 to 12 months. ${ }^{6} \quad 9 \quad 10 \quad 29 \quad 34-36$ Furthermore, the majority of studies have utilised cross-sectional design which lacks information about temporality.

To the best of our knowledge, this is the first longitudinal study with adolescents to examine the relationship between CPF and self-reported neurological symptoms. In this study, a gradual increase in neurological symptoms, relative to the baseline time interval, was observed among the applicators from during the CPF application period after accounting for the number of days worked during the week, home use of pesticides by the participant, age, education and family monthly income levels. A significant 30 percentage point increase in the neurological symptoms relative to the baseline time interval was observed on time interval 8 (days $63-77$ of the study). This is perhaps due to a second short CPF application episode in the same season in field station 1. Self-reported symptoms among applicators remained significantly elevated from the preapplication period until day 217, approximately 5 months after the cessation of exposure showing evidence that despite discontinuation of CPF application, repeated exposure of this pesticide led to persistence of neurological health effects for several months. Compared with the applicators, the non-applicators showed relatively late reporting of neurological symptoms, perhaps due to the environmental CPF exposure. It is interesting to note that the non-applicators still reported approximately nine percentage point more symptoms relative to baseline at the last time interval (days 105-217). Residual CPF can survive in indoor environments for an extended period of time; it can also rapidly bind to soil and plants and has a half-life of several months in soil. ${ }^{37} 38$ We anticipate that because of these properties, CPF remained in the environment as a potential source of environmental exposure leading to increased symptom reporting among non-applicators.

Symptom reporting over time showed a recovery phase at time interval 10 (days 105-207) when the percentage of symptom reporting relative to baseline declined substantially from the previous time intervals (table 3, figure 2). Using the same sample, we recently demonstrated that the applicators and non-applicators experienced peak median BChE depression during the CPF application period, but BChE returned to the baseline level by the end of the study (day 217/5 January 2011) ${ }^{32}$ We anticipate that symptoms were following the 
Table 4 Summary of regression analysis for biomarkers of exposure and effect of chlorpyrifos predicting average percentage of neurological symptoms over the entire study stratified by applicator status

\begin{tabular}{|c|c|c|c|c|c|c|}
\hline \multirow[b]{2}{*}{ Explanatory variables } & \multicolumn{3}{|c|}{ Unadjusted models } & \multicolumn{3}{|c|}{ Adjusted models* } \\
\hline & B (se) & $95 \% \mathrm{Cl}$ & p Value & B (se) & $95 \% \mathrm{Cl}$ & p Value \\
\hline \multicolumn{7}{|l|}{ For non-applicators } \\
\hline Ln TCPy (mg/g Cr) $(\mathrm{n}=28)$ & $0.29(0.76)$ & -1.26 to 1.84 & 0.71 & $0.57(0.79)$ & -1.06 to 2.20 & 0.47 \\
\hline Ln (Post-AChE/Pre-AChE) $(n=21)$ & $-1.25(16.41)$ & -35.59 to 33.1 & 0.94 & $-6.57(18.80)$ & -43.64 to 33.50 & 0.73 \\
\hline Ln (Post-BChE/Pre-BChE) $(n=21)$ & $2.23(7.04)$ & -12.51 to 16.98 & 0.76 & $2.50(7.63)$ & -13.77 to 18.77 & 0.75 \\
\hline \multicolumn{7}{|l|}{ For applicators } \\
\hline Ln TCPy (mg/g Cr) $(\mathrm{n}=42)$ & $4.56(0.63)$ & 3.29 to 5.84 & $<0.001$ & $2.68(0.93)$ & 0.78 to 4.57 & 0.007 \\
\hline Ln (Post-AChE/Pre-AChE) $(n=28)$ & $-24.21(12.79)$ & -50.50 to 2.09 & 0.07 & $-11.60(12.44)$ & -37.46 to 14.25 & 0.36 \\
\hline Ln (Post-BChE/Pre-BChE) $(n=29)$ & $-14.52(4.61)$ & -23.97 to -5.07 & 0.004 & $-7.33(5.93)$ & -19.63 to 4.97 & 0.23 \\
\hline
\end{tabular}

BChE activity pattern, that is, as the BChE activity was returning to the baseline level, neurological symptoms were going through the recovery phase.

Prior to this study, a cross-sectional study on Egyptian cotton field workers reported associations between OP exposure and neurological symptoms..$^{20}$ Similar to an Indian study on occupationally exposed adolescents, ${ }^{22}$ the previous Egyptian adolescent study ${ }^{20}{ }^{21}$ presented descriptive statistics to show the difference between exposed and unexposed adolescents in terms of the prevalence of various neurological symptoms. However, these studies did not take potential sociodemographic confounders into account. Results of the present study were consistent with several longitudinal studies conducted in adult populations. In one study of occupationally and non-occupationally OP pesticide-exposed farmers and fishermen, delayed persistence of neurological symptoms were found during the 2-year follow-up. ${ }^{7}$ Results from a clinical examination of the same cohort found that there were deficits related to sensory function. ${ }^{39}$ Another study, conducted over 3 years with Colorado farm workers, reported an association between OP exposure and symptoms of depression. ${ }^{12}$ Consistency in the results across studies indicates that a Q16 based self-reported questionnaire used in all of these studies is a reliable measure to estimate health effects resulting from OP (in this case CPF) exposure.

Our study is also novel in its approach of including prospective measures of biomarkers. First, instead of using single-time point biomarker data commonly used in cross-sectional studies, our study analysed urinary TCPy levels at multiple time points. The collection of pre, during and post application samples resulted in a precise estimate of cumulative exposure from 11 April to 5 January. ${ }^{32}$ This has enabled us to overcome a historical challenge in characterising OP exposure and allows us to subsequently examine the association of cumulative exposure with neurological symptoms. An additional limitation often encountered by past studies was the absence of established baseline AChE and BChE levels.
A recent adult study examining the variation of $\mathrm{ChE}$ levels among OP pesticides and carbamate-exposed fieldworkers could not establish any baseline AChE/ BChE due to the mobility of the migrant study population. ${ }^{40}$ Another Egyptian adolescent study also reported a greater reduction of $\mathrm{AChE}$ activity among pesticide applicators compared to controls. ${ }^{20} 21$ By collecting blood samples prior to the start of the application season, baseline data were established, which allowed us to compute more precise measures of change in activities of AChE and BChE from pre-exposure to postexposure periods.

Two previous studies of Kenyan and Palestinian farm workers, which measured ChE levels before and after exposure, found associations between $\mathrm{ChE}$ inhibition and respiratory, eye and neurological symptoms. ${ }^{9} 41$ Potential occupational confounding factors (eg, residential application of pesticides and number of days worked in agriculture) that are associated with neurological symptoms ${ }^{20}{ }^{42}$ were not taken into account while examining exposure-outcome associations in these previous studies. These potential confounding variables were included in our study questionnaires and later examined during statistical analysis.

We identified a comparison group (of nonapplicators) who were similar in demographic characteristics to our applicators. It is often true that control groups in occupational settings may not be truly unexposed. ${ }^{1}$ In our study, close proximity to the agricultural field (less than $25 \mathrm{~m}$ ) and application of pesticides at home were the two environmental factors offering potential exposure opportunities to the non-applicators as indicated by elevated urinary TCPy levels during the period of CPF application to cotton fields. ${ }^{32}$ To encounter this potential confounder, all statistical models were adjusted for these two variables in addition to other sociodemographic variables.

It is difficult to explain why we found no relationship between TCPy and neurological symptoms among the non-applicators when a delayed effect of environmental or 


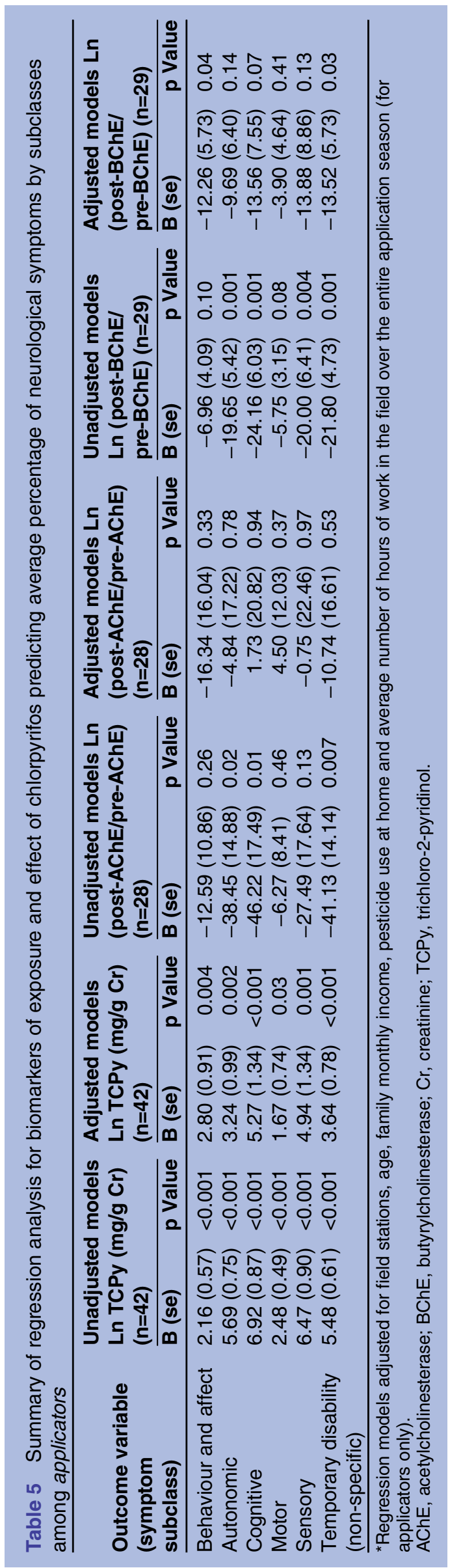

passive CPF exposure on symptoms was evident among this subgroup in the corresponding GEE model (table 3 and figure 2). One possible explanation is that the range of cumulative exposure was much lower among the nonapplicators (154-24 $180 \mathrm{mg} / \mathrm{g}$ creatinine; median $2591 \mathrm{mg} / \mathrm{g}$ creatinine) compared to the applicators (232$28260 \mathrm{mg} / \mathrm{g}$ creatinine; median $10318 \mathrm{mg} / \mathrm{g}$ creatinine). The small sample size and differences in cumulative exposure might have contributed to the non-significant association in the non-applicator subgroup. Some other undocumented environmental factors such as working during high temperatures, along with carrying a heavy backpack during CPF application, might have positively confounded the association among the applicators.

We acknowledge that we relied on a self-reported outcome measure. Therefore, there was a possibility that the frequent completion of the neurological symptoms survey (32 times over 8 months) could itself have had an influence on the increase in symptoms during the CPF application season. This could partially explain why these symptoms were not associated with TCPy levels among non-applicators.

The non-specific nature of many of the symptoms is another limitation of the current study; the biological significance of these self-reported symptoms is unknown. However, the goal of the study was not to establish that more symptoms lead to the development of any neurological disease. Rather, we attempted to examine how repeated or cumulative exposure to CPF determined the pattern of neurological symptoms over the entire season. Five of the symptoms included in our questionnaire are considered non-specific, including headache, dizziness, fatigue, loss of consciousness and insomnia. The remaining 20 symptoms were classified into more specific neurological functions such as behaviour, autonomic, sensory, cognitive or motor functions. When we excluded these non-specific symptoms from the summary measure, the estimated $\beta$ s for the associations of TCPy and change in $\mathrm{AChE}$ and BChE activities with average percentage of 20 neurological symptoms were found to be $3.19(\mathrm{p}<0.001)$, $-6.11(\mathrm{p}=0.60)$ and $-9.49(\mathrm{p}=0.05)$, respectively, after accounting for potential covariates.

\section{CONCLUSION}

Our study reinforces the need for the development and execution of intervention programmes for the residents of agricultural communities, including pesticide applicators, in developing countries. Future interventions should include hygiene practices, behaviours and use of protective equipment in occupational and residential environments. Our study is the first to demonstrate that repeated occupational CPF exposure is an important determinant of neurological symptoms in adolescent applicators and non-applicators over time, with symptoms peaking during the exposure season and partly recovering in the months following exposure. The study also showed a significant association between cumulative 
CPF exposure and symptoms, using cumulative urinary TCPy as a biomarker of exposure. Future studies are needed to assess the temporal and dose-dependent effects of repeated CPF exposure on neurological symptoms and neurobehavioral deficits in children, adolescents and adults to identify the most sensitive populations.

\section{Author affiliations}

${ }^{1}$ Occupational and Environmental Health, College of Public Health, University of lowa, lowa City, Iowa, USA

${ }^{2}$ Community Medicine and Public Health Department, Faculty of Medicine, Menoufia University, Shebin El-Kom, Egypt

${ }^{3}$ Department of Social and Preventative Medicine, State University of New York, Buffalo, New York, USA

${ }^{4}$ Center for Research on Occupational and Environmental Toxicology, Oregon Health and Science University, Portland, Oregon, USA

${ }^{5}$ Clinical Pathology and Hematology and Immunology, Menoufia University, Shebin El-Kom, Egypt

${ }^{6}$ Department of Pharmacology and Toxicology, State University of New York, Buffalo, New York, USA

Acknowledgements The authors would like to thank the Egyptian Ministry of Agriculture and the adolescents and their parents for their participation, Steve Hutton (Dow Agrosciences, Indianapolis, Indiana, USA) for providing 13C-15N-3,5,6-TCPy, Barbara McGarrigle for the urinary trichloro-2-pyridinol analytical work and the Research Team at Menoufia University.

Contributors All authors have made substantial contributions to the conception and design, acquisition of data or analysis and interpretation of data in the study; KK has taken the lead to draft the manuscript. Whereas other authors have revised the draft critically for important intellectual content. All of the authors have provided final approval of the version to be published. DSR is the Principal Investigator of the study and has supervised each step of the manuscript development process. She has been listed as the corresponding author.

Funding The work was supported by the Fogarty International Center and the National Institute of Environmental Health Sciences (NIEHS, grant \#ES017223).

\section{Competing interests None.}

Patient consent Obtained.

Ethics approval The study was approved by the Oregon Health and Science University institutional review board (IRB) in June 2009, and by the Medical Ethics Committee of the Faculty of Medicine, Menoufia University in July 2009.

\section{Provenance and peer review Not commissioned; externally peer reviewed.}

Data sharing statement Additional unpublished data from the study are available to DSR (diane-rohlman@uiowa.edu), AAI (aa-ismail@hotmail.com) and JR0 (aa-ismail@hotmail.com) in Excel or SPSS datasets. They can be reached by email.

Open Access This is an Open Access article distributed in accordance with the Creative Commons Attribution Non Commercial (CC BY-NC 3.0) license, which permits others to distribute, remix, adapt, build upon this work noncommercially, and license their derivative works on different terms, provided the original work is properly cited and the use is non-commercial. See: http:// creativecommons.org/licenses/by-nc/3.0/

\section{REFERENCES}

1. Kamel F, Hoppin JA. Association of pesticide exposure with neurologic dysfunction and disease. Environ Health Perspect 2004;112:950-8.

2. Solomon C, Poole J, Palmer KT, et al. Acute symptoms following work with pesticides. Occup Med (Lond) 2007;57:505-11.

3. Wesseling C, van Wendel de Joode B, Keifer M, et al. Symptoms of psychological distress and suicidal ideation among banana workers with a history of poisoning by organophosphate or n-methyl carbamate pesticides. Occup Environ Med 2010;67:778-84.

4. Alavanja MC, Hoppin JA, Kamel F. Health effects of chronic pesticide exposure: cancer and neurotoxicity. Annu Rev Public Health 2004;25:155-97.

5. Beseler C, Stallones L. Safety practices, neurological symptoms, and pesticide poisoning. J Occup Environ Med 2003; 45:1079-86

6. London L, Nell V, Thompson ML, et al. Effects of long-term organophosphate exposures on neurological symptoms, vibration sense and tremor among South African farm workers. Scand J Work Environ Health 1998;24:18-29.

7. Delgado E, McConnell R, Miranda J, et al. Central nervous system effects of acute organophosphate poisoning in a two-year follow-up. Scand J Work Environ Health 2004;30:362-70.

8. Keifer M, Rivas F, Moon JD, et al. Symptoms and cholinesterase activity among rural residents living near cotton fields in Nicaragua. Occup Environ Med 1996;53:726-9.

9. Ohayo-Mitoko GJ, Kromhout H, Simwa JM, et al. Self reported symptoms and inhibition of acetylcholinesterase activity among Kenyan agricultural workers. Occup Environ Med 2000;57:195-200.

10. Smit LA, van-Wendel-de-Joode BN, Heederik D, et al. Neurological symptoms among Sri Lankan farmers occupationally exposed to acetylcholinesterase-inhibiting insecticides. $\mathrm{Am} J$ Ind Med 2003;44:254-64.

11. Farahat FM, Rohlman DS, Storzbach D, et al. Measures of short-term test-retest reliability of computerized neurobehavioral tests. Neurotoxicology 2003;24:513-21.

12. Beseler $\mathrm{CL}$, Stallones $\mathrm{L}$. A cohort study of pesticide poisoning and depression in Colorado farm residents. Ann Epidemiol 2008;18:768-74.

13. McCauley LA, Shapiro SE, Scherer JA, et al. Assessing pesticide safety knowledge among Hispanic migrant farmworkers in Oregon. J Agric Saf Health 2004;10:177-86.

14. McCauley LA, Sticker D, Bryan C, et al. Pesticide knowledge and risk perception among adolescent Latino farmworkers. J Agric Saf Health 2002;8:397-409.

15. London L, Beseler C, Bouchard MF, et al. Neurobehavioral and neurodevelopmental effects of pesticide exposures. Neurotoxicology 2012;33:887-96.

16. Costa LG, Li WF, Richter RJ, et al. The role of paraoxonase (PON1) in the detoxication of organophosphates and its human polymorphism. Chem Biol Interact 1999;119-120:429-38.

17. Costa LG, Cole TB, Vitalone A, et al. Measurement of paraoxonase (PON1) status as a potential biomarker of susceptibility to organophosphate toxicity. Clin Chim Acta 2005;352:37-47.

18. Rauh VA, Perera FP, Horton MK, et al. Brain anomalies in children exposed prenatally to a common organophosphate pesticide. Proc Natl Acad Sci USA 2012;109:7871-6.

19. Ecobichon DJ. Pesticide use in developing countries. Toxicology 2001;160:27-33.

20. Abdel Rasoul GM, Abou Salem ME, Mechael AA, et al. Effects of occupational pesticide exposure on children applying pesticides. Neurotoxicology 2008;29:833-8.

21. Ismail A, Rohlman D, Rasoul GA, et al. Clinical and biochemica parameters of children and adolescents applying pesticides. Int $J$ Occup Environ Med 2010;1:132-43.

22. Rastogi SK, Tripathi S, Ravishanker D. A study of neurologic symptoms on exposure to organophosphate pesticides in the children of agricultural workers. Indian J Occup Environ Med 2010;14:54-7.

23. Nolan RJ, Rick DL, Freshour NL, et al. Chlorpyrifos: pharmacokinetics in human volunteers. Toxicol Appl Pharmacol 1984;73:8-15

24. Farahat FM, Ellison CA, Bonner MR, et al. Biomarkers of chlorpyrifos exposure and effect in Egyptian cotton field workers. Environ Health Perspect 2011;119:801-6.

25. Egeghy PP, Cohen Hubal EA, Tulve NS, et al. Review of pesticide urinary biomarker measurements from selected US EPA children's observational exposure studies. Int $J$ Environ Res Public Health 2011;8:1727-54.

26. Hofmann JN, Keifer MC, De Roos AJ, et al. Occupational determinants of serum cholinesterase inhibition among organophosphate-exposed agricultural pesticide handlers in Washington State. Occup Environ Med 2010;67:375-86.

27. Fenske RA, Farahat FM, Galvin K, et al. Contributions of inhalation and dermal exposure to chlorpyrifos dose in Egyptian cotton field workers. Int J Occup Environ Health 2012;18:198-209.

28. Lundberg I, Hogberg M, Michelsen $\mathrm{H}$, et al. Evaluation of the Q16 questionnaire on neurotoxic symptoms and a review of its use. Occup Environ Med 1997;54:343-50. 
29. Kamel F, Engel LS, Gladen BC, et al. Neurologic symptoms in licensed pesticide applicators in the Agricultural Health Study. Hum Exp Toxicol 2007;26:243-50.

30. Fabiny DL, Ertingshausen G. Automated reaction-rate method for determination of serum creatinine with the CentrifiChem. Clin Chem 1971;17:696-700.

31. Liang K-Y, Zeger SL. Longitudinal data analysis using generalized linear models. Biometrika 1986;73:13-22.

32. Crane AL, Abdel Rasoul G, Ismail A, et al. Longitudinal assessment of chlorpyrifos exposure and effect biomarkers in adolescent Egyptian agricultural workers. J Expo Sci Environ Epidemiol 2013;23:356-62.

33. Stallones L, Beseler C. Pesticide illness, farm practices, and neurological symptoms among farm residents in Colorado. Environ Res 2002;90:89-97.

34. Gomes J, Lloyd O, Revitt MD, et al. Morbidity among farm workers in a desert country in relation to long-term exposure to pesticides. Scand J Work Environ Health 1998;24:213-19.

35. Pilkington A, Buchanan D, Jamal GA, et al. An epidemiological study of the relations between exposure to organophosphate pesticides and indices of chronic peripheral neuropathy and neuropsychological abnormalities in sheep farmers and dippers. Occup Environ Med 2001;58:702-10.
36. Steenland K, Dick RB, Howell RJ, et al. Neurologic function among termiticide applicators exposed to chlorpyrifos. Environ Health Perspect 2000;108:293-300.

37. CDC. National report on human exposure to environmental chemicals (Centers for Disease Control and Prevention) 2009. http:// www.cdc.gov/exposurereport (accessed 18 Feb 2012)

38. Eaton DL, Daroff RB, Autrup H, et al. Review of the toxicology of chlorpyrifos with an emphasis on human exposure and neurodevelopment. Crit Rev Toxicol 2008;38(Suppl 2):1-125.

39. Miranda J, McConnell R, Wesseling C, et al. Muscular strength and vibration thresholds during two years after acute poisoning with organophosphate insecticides. Occup Environ Med 2004;61:e4.

40. Quandt SA, Chen H, Grzywacz JG, et al. Cholinesterase depression and its association with pesticide exposure across the agricultural season among Latino farmworkers in North Carolina. Environ Health Perspect 2010;118:635-9.

41. Safi JM, Abu Mourad TA, Yassin MM. Hematological biomarkers in farm workers exposed to organophosphorus pesticides in the Gaza Strip. Arch Environ Occup Health 2005; 60:235-41.

42. Kamel F, Engel LS, Gladen BC, et al. Neurologic symptoms in licensed private pesticide applicators in the agricultural health study. Environ Health Perspect 2005;113:877-82. 